\title{
Etapas del proceso de gestión de mantenimiento. Una experiencia en la industria petrolera venezolana
}

\author{
Stages of the maintenance management process. An experience in the
} Venezuelan oil industry

\section{Jorge Antunez}

jorgelantunez@hotmail.com

Código ORCID: 0000-0002-5458-8087

Universidad del Zulia. Maracaibo, Venezuela
4 Articulo recibido en julio 2019

< Arbitrado en agosto 2019

< Publicado en enero 2020

Resumen

La gestión de mantenimiento se ha convertido en una herramienta necesaria en las organizaciones, por lo tanto, en este artículo se describen las etapas del proceso de gestión de mantenimiento en la industria petrolera venezolana, bajo una metodología descriptivo, con diseño no experimental, transeccional y de campo. La población quedo conformada por gerentes, líderes y supervisores de las empresas que realizan el mantenimiento a las estructuras metálicas de la industria petrolera región occidente. A objeto de recolectar la información se aplicó un cuestionario de 30 ítems, con escala de frecuencia. El mismo fue validado por el juicio de expertos. Y para calcular su confiabilidad se utilizó el coeficiente Alfa de Cronbach, obteniéndose 0,82. El análisis de los resultados se realizó a través de las frecuencias. Se concluye que estas organizaciones poseen alta presencia de estas etapas en su gestión, siendo estas la planificación, organización, programación, ejecución, control y evaluación.

Palabras clave: Control, ejecución, etapas del proceso de gestión de mantenimiento, evaluación, organización, planificación, programación

\begin{abstract}
Maintenance management has become a necessary tool in organizations, therefore, this article describes the stages of the maintenance management process in the Venezuelan oil industry, under a descriptive methodology, with a non-experimental, transectional and field. The population was made up of managers, leaders and supervisors of the companies that perform maintenance on the metal structures of the oil industry in the western region. In order to collect the information, a 30-item questionnaire was applied, with a frequency scale. It was validated by expert judgment. And to calculate its reliability, the Cronbach's alpha coefficient was used, obtaining 0.82 . The analysis of the results was carried out through the frequencies. It is concluded that these organizations have a high presence of these stages in their management, being these the planning, organization, programming, execution, control and evaluation.
\end{abstract}

Keywords: Control, execution, stages of the maintenance management process, evaluation, organization, planning, programming 


\section{INTRODUCCIÓN}

En el mundo actual, el mantenimiento se presenta como sinónimo de buena gestión empresarial, pues su propósito es apoyar la producción y contribuir al logro de los objetivos organizacionales, disminuyendo los costos para aumentar así los beneficios de la organización. Dentro de este contexto, la necesidad de que las empresas sean eficientes en su gestión de mantenimiento, como una condición necesaria para poder competir en el mercado, ha llevado a que éstas ejecuten acciones tendentes a atacar las causas de sus debilidades.

Desde esta perspectiva, la práctica de una adecuada gestión de mantenimiento permitirá a las organizaciones reestructurar su funcionamiento, haciendo posible eliminar muchos de los problemas que causan las fallas, ineficiencias, la baja productividad y la pobre competitividad. Desde una visión amplia, la gestión de mantenimiento constituye una parte importante del enfoque integral que toda administración debe manejar, en efecto, sus mediciones la clave para la gestión de sus procesos operativos permitiendo alcanzar los objetivos organizacionales, convirtiéndose en una herramienta para el sistema de la toma de decisiones.

En este marco de referencia, la gestión de mantenimiento se concibe a criterio de Becerra (2005), como un parámetro de referencia para evaluar, a través, de la supervisión de la planificación, ejecución y control, el conjunto de actividades propias de la función, que permiten el uso efectivo y eficaz de los recursos con que cuenta la organización, para alcanzar los objetivos que satisfacen los requerimientos de los diferentes grupos de interés, cuyo objetivo básico consiste en incrementar la disponibilidad de los Sistemas Productivos (SP), partiendo de la ejecución de los mismos, mediante las mejoras incrementales a bajo costo, para ser competitivo, logrando que funcionen de forma eficiente y confiable dentro de un contexto de operación.

Bajo estas premisas, esta investigación estuvo direccionada a describir las tapas del proceso de gestión de mantenimiento en las empresas que realizan el mantenimiento a las estructuras metálicas de la industria petrolera en occidente, siendo ellas: IMDISMA C.A, SERTEJERCA y ANCRI RADIADORES C.A; las cuales se encargan de satisfacer la demanda de mantenimiento a las estructuras reseñadas, siendo su mayor cliente el sector petrolero, en este caso la industria petrolera venezolana, Petróleos de Venezuela S.A. (PDVSA). Frente a un cliente tan exigente, es importante que estas empresas puedan contar con una eficiente gestión de mantenimiento para satisfacer las expectativas de sus clientes.

\section{Etapas del proceso de gestión de mantenimiento}

El proceso de gestión de mantenimiento, como explica Omeñaca (2009), comprende una técnica de gestión que sirve para analizar, documentar y disminuir los riesgos asociados a aquello que se está estudiando; se utiliza principalmente en la mejora de procesos productivos y productos con la intención de evitar la aparición de problemas de calidad, aunque también se utiliza en otros ámbitos de la gestión, por ejemplo, en la prevención de riesgos laborales.

Su campo de aplicación comprende todas aquellas situaciones de operación o producción en las que sea necesario planificar o re-planificar productos, servicios o procesos: producto o servicio que sirve como 
herramienta de optimización; el proceso que permite la obtención del producto o la prestación del servicio y sirve como herramienta de optimización antes de su traspaso.

En general, como señala Omeñaca (2009), el proceso para la ejecución de una gestión de mantenimiento es lineal, se desarrolla en seis (6) fases: planificación, organización, programación, ejecución, control y evaluación. Mientras que, para García (2009) el proceso de gestión del mantenimiento conforma el conjunto de tareas realizadas por el usuario para mantener la funcionabilidad del sistema durante su vida operativa. Así, conociendo el significado del término proceso, puede inferirse que este proceso está compuesto por una serie de etapas que deberán considerarse para aplicarlo, y éstas deberán estar estrechamente vinculadas unas con otras, generando retroalimentación de la información que se genera en cada una de ellas.

En este orden de ideas, Márquez (2010) define las e tapas del proceso de la gestión de mantenimiento como el trabajo de planificar, organizar, programar, ejecutar, controlar y evaluar tomando en consideración los recursos, estas etapas deben realizarse para maximizar tanto la disponibilidad como efectividad de la infraestructura requerida por el sistema de producción. De acuerdo a este autor, el propósito de las etapas del proceso es mejorar la funcionalidad de los componentes de equipos o maquinarias en función de la filosofía de las empresas en cuanto a costos, calidad adecuada, momento oportuno, lugar apropiado; con la adopción de medidas, así como, la realización de acciones que permitan alcanzar los objetivos y la efectividad de sus procesos.
Ahora bien, autores como Martínez (2007) afirman que, para cumplir las etapas de este proceso es prioritario definir un sistema de elementos con base a la dirección, que se relacione con la planificación, organización, programación, ejecución, control y evaluación. Bajo esta óptica la gestión de mantenimiento es un ciclo que se da en forma secuencial, cuyo proceso consta de seis (6) etapas, ya mencionadas, y este ciclo permite realizar una retroalimentación a fin de permitir una mejora continua.

Con base en las definiciones antes señaladas, para efectos de esta investigación, se definen las etapas del proceso de la gestión de mantenimiento como el conjunto de acciones pertinentes para su cumplimiento, que contribuyan a la efectividad de los procesos productivos, y considerada dentro de la tendencia sistémica de la gestión de mantenimiento como: planificación, organización, programación, ejecución, control y evaluación, tomando en cuenta siempre los recursos. A continuación se pasa a explicar cada una de estas etapas, que conforman los indicadores de esta dimensión.

\section{Planificación}

En el contexto específico del mantenimiento, la planificación está referida a determinar los recursos necesarios para satisfacer la demanda de trabajos a los que va a responder el mantenimiento; es el proceso a través del cual se determinan qué elementos serán necesarios para realizar una tarea de mantenimiento, antes que se dé inicio al trabajo (Leal y Zambrano, 2011). Entre los recursos necesarios, se incluyen la fuerza laboral, los repuestos, el equipo y las herramientas. 
Desde esta perspectiva, Clemenza (2010) indica que, en la planificación es fundamental tomar en cuenta aspectos como procedimientos, manuales, mano de obra, materiales, partes, repuestos, equipos $y$ herramientas de soporte. Al profundizar en cada uno de ellos, resultan las siguientes interrogantes:

$\checkmark$ Procedimientos: ¿̇qué se va a hacer?, ¿̇cómo se va a hacer? ¿̇con qué seguridad se hará?, ¿̇con cuál procedimiento técnico?, ¿̇con cuál manual?

$\checkmark$ Mano de obra: ¿̇qué tipo y cantidad?, ¿̇cuánto tiempo por tipo?, ¿̇cuánto tiempo requerirá?, ¿̇con qué secuencia se utilizará?

$\checkmark$ Materiales, partes y repuestos: ¿̇qué tipo de insumos?, ¿̇qué cantidad?, ¿̇cuál tipo?

$\checkmark$ Equipos: ¿̇cuáles herramientas se requerirán?, ¿̇cuáles equipos de soporte?, ¿̇por cuánto tiempo?

Señala el autor, que el éxito en la gestión de mantenimiento depende del alcance de los objetivos previstos en la planificación, detallados como: alcanzar el mayor dinamismo y flexibilidad de la organización de mantenimiento; brindar información oportuna y confiable a la organización a fin de lograr medir la gestión; planificar la mayor cantidad posible de trabajos de mantenimiento; diseñar los planes de mantenimiento; colaborar con la elaboración del presupuesto anual de mantenimiento; diseñar procedimientos técnicos; y contribuir en la elaboración de planes de servicio preventivo a equipos.

En este marco referencial, para Duffuaa $y$ otros (2010), la planificación del mantenimiento se refiere al proceso mediante el cual se determinan y preparan todos los elementos requeridos para efectuar una tarea antes de iniciar un trabajo. El proceso de planeación comprende todas las funciones relacionadas con la preparación de la orden de trabajo, lista de materiales, la requisición de compra, la mano de obra a utilizar, los estándares de tiempo y todos los datos necesarios antes de programar y liberar la orden de trabajo. Por lo que, la planificación de mantenimiento es el desarrollo sistemático de programas de acción, encaminados hacia los objetivos de mantenimiento establecidos a priori, mediante el análisis, evaluación y selección entre alternativas previstas, que permitan la coordinación de elementos productivos del mantenimiento a fin de obtener resultados en cantidad, calidad, tiempo y costos deseados.

Antes de iniciar el proceso de planeación de las actividades de mantenimiento, según lo indican Zambrano y Leal (2006), se deben conocer los siguientes aspectos:

$\checkmark$ Inventario de equipos: se refiere a la lista de equipos que posee toda organización y que requieren acciones de mantenimiento.

$\checkmark$ Inventario de recursos de mantenimiento: lista de materiales, repuestos, equipos, herramientas e instrumentos necesarios para la ejecución de las diferentes labores de mantenimiento.

$\checkmark$ Sistema de codificación: código con el que se identifica un equipo. El código es una representación alfanumérica que debe ser de fácil interpretación por parte de todos los usuarios del departamento de mantenimiento y empresa en general.

$\checkmark$ Registro de información técnica: planilla que recopila toda la información descriptiva del objeto a mantener. En esta ficha técnica se resaltan las características más importantes de un objeto en 
específico, a fin de tener un mayor conocimiento de éste y facilitar su ubicación ante la presencia de fallas o acciones de mantenimiento.

$\checkmark$ Orden de trabajo: formato donde se detallan las instrucciones escritas para el trabajo que se va a realizar y debe ser llenada para todos los trabajos. El propósito del sistema de órdenes de trabajo, según Duffuaa y otros (2010), es proporcionar medios para: solicitar por escrito el trabajo que se va a realizar; asignar el mejor método y los trabajadores más calificados para el trabajo; reducir el costo mediante la utilización eficaz de los recursos( mano de obra y material); mejorar la planificación y programación de las actividades; mantener y controlar el trabajo y mejorar el mantenimiento en general mediante los datos recopilados en la orden de trabajo, las cuales serán utilizadas para el control y programas de mejora continua.

Ahora bien, otro aspecto esencial durante la planificación lo constituye el pronóstico de la carga de mantenimiento, ya que permite determinar el nivel deseado de eficacia y utilización de recursos. Duffuaa y otros (2010), definen este pronóstico como el proceso mediante el cual se predice la carga de mantenimiento. En una planta esta carga varía aleatoriamente $y$, entre otros factores, puede ser una función de la edad del equipo, el nivel de su uso, la calidad de mantenimiento, factores climáticos y las destrezas de los trabajadores de mantenimiento.

Para el investigador, planificar es decidir con anticipación el cómo hacer, el qué hacer, cuándo hacerlo, y quién debe hacerlo, esto con el $f$ in de contribuir al logro de los objetivos de la organización, considerando su visión para seleccionar las estrategias a seguir. Planear, es la base para poder llevar a cabo las acciones de mantenimiento, sin importar de que tipo sea el mismo, así tener de una manera ordenada los pasos a seguir, para que se cumpla el trabajo en sinergia. Su objetivo primordial debe ser lograr con el mínimo coste el mayor tiempo en servicio de los recursos que intervienen en la organización, con el fin de conseguir la máxima disponibilidad, aportando la mayor productividad y seguridad de funcionamiento.

\section{Organización}

En el contexto del mantenimiento, Duffuaa y otros (2010) afirman que, la organización del mantenimiento consiste en definir cómo está estructurado el mismo, bien sea por departamentos, área o de manera centralizada. Mientras que, García (2009) define la organización como la etapa donde se debe determinar la estructura de trabajo, las funciones dentro de la estructura, las relaciones externas e internas, los procedimientos para el flujo y registro de información, así como la preparación de todos los recursos involucrados en el proceso: materiales, humanos, tecnológicos, entre otros.

En este contexto, Jiménez y Milano (2006) afirman que, en la organización se deben agrupar a las personas con base a las experiencias comunes, representando una especialización funcional, en el caso del mantenimiento la organización se puede distribuir en planificación, control, ingeniería, estudio de fallas, control, entre otros.

Por su parte, Martínez (2007) señala que, organizar es estructurar, dar forma e interrelacionar las partes de un complejo previamente planeado, disponiendo de los 
recursos de la empresa (hombre, maquinas, materiales, entre otros) de tal forma que esta pueda funcionar según lo previsto en la planeación, tomando en cuenta los siguientes factores: puestos, hombres, autoridad y responsabilidad. Agrega Martínez (2007) que, la organización del mantenimiento se vale de dos fases:

$\checkmark$ La fase organizativa en la que se determina la estructura de trabajo, las funciones dentro de la estructura, las relaciones externas e internas, los procedimientos para el flujo, registro de información y documentación.

$\checkmark$ La fase preparatoria donde se define la preparación de los recursos materiales, humanos, la documentación, las instalaciones, entre otros.

En este marco de referencia, afirma García (2009), el cumplimiento de un plan requiere de organización de cada una de las etapas, también se le llama a la organización mantenimiento planificado, el cual debería tener lugar antes de que ocurra un fallo o una avería, se efectúa bajo condiciones controladas sin la existencia de algún error en el sistema. Se realiza a razón de la experiencia y pericia del personal a cargo, los cuales son los encargados de determinar el momento necesario para llevar a cabo dicho procedimiento. Adicionalmente, el fabricante también puede estipular el momento adecuado a través de los manuales técnicos.

A juicio del investigador la organización, en la gestión de mantenimiento, es la fase de procura y distribución de responsabilidades, fijación de tiempo, esfuerzo, costo, necesarios para cada una de las actividades planificadas. Así, el mantenimiento debe organizarse para dar respuesta rápida, efectiva, cónsona con la realidad organizativa.

\section{Programación}

El objetivo de la programación de mantenimiento, según lo señalan Duffuaa y otros (2010), consiste en señalar cuando se deben realizar las diferentes instrucciones técnicas de cada objeto de mantenimiento. La programación puede ser para periodo anual, semestral, mensual, semanal $\circ$ diario, dependiendo de la dinámica del proceso y del conjunto de actividades a programar.

En el caso de la planificación de mantenimiento programado, los programas cubren períodos de un año (generalmente). Este tipo de programa es ejecutado por el personal de la organización de mantenimiento o por entes foráneos, en el caso de actividades cuya ejecución es por contrato los tipos de frecuencia más comunes son quincenal, mensual, bimensual, trimestral, semestral, y anual (Norma COVENIN 3049-93).

En el caso del mantenimiento rutinario, los programas cubren hasta periodos de una semana ya que están compuestos por instrucciones simples que deben ser ejecutados por el mismo operario, dichas instrucciones las porta el operario en su carpeta de trabajo o son adheridas al objeto a mantener o son colocadas en una cartelera próxima a una serie de objetos, sus frecuencias comunes son: cada hora de trabajo, cada pieza producida, cada turno, diario, inter-diario, cada día y semanal.

Por otra parte, según Leal y Zambrano (2011), la programación del trabajo encomienda al personal de mantenimiento las asignaciones específicas de trabajo, con la suficiente anticipación como para garantizar la máxima coordinación entre mano de obra, 
material y equipo. La programación es un plan de acción cuidadosamente preparado que ha considerado la disponibilidad de mano de obra, materiales, equipos y las prioridades relativas de los trabajos. Los principios más importantes de la programación efectiva de mantenimiento son los siguientes, según lo acotan estos autores:

$\checkmark$ Los programas deberán estar basados en lo que sea más probable que suceda, no en lo que nos gustaría que sucediera.

$\checkmark$ Debe esperarse una revisión del programa. Debe existir flexibilidad en el sistema, de tal forma que puedan enfrentarse las interrupciones de último minuto.

$\checkmark$ El programa es un medio para lograr un fin, no un fin en sí mismo. Es muy fácil que el entusiasmo del personal les haga perder de vista el objetivo de un buen programa de trabajo: proporcionar al cliente un servicio con mayor calidad al precio más bajo.

La persona responsable de la planeación de mantenimiento, de acuerdo a lo postulado por Duffuaa y otros (2010), debe tener varios elementos antes de iniciar el programa de trabajo: orden de trabajo, plan de trabajo (para trabajo correctivo mayor), acumulación de órdenes de trabajo pendientes, reporte de disponibilidad de mano de obra y materiales, programa maestro de mantenimiento preventivo y programa de producción.

La programación de mantenimiento es un procedimiento que inicia con los trabajos que tengan la prioridad más alta en la enumeración de pendientes. El trabajo correctivo mayor disponible, significa un trabajo que se ha estimado por completo y para el cual se ha elaborado un plan. Las órdenes de trabajo se toman de la enumeración pendiente $y$ se agregan al programa hasta que se utilice todo el tiempo disponible. Si la prioridad de órdenes de trabajo así lo exige, debe solicitarse tiempo extra para cumplir el programa.

Las herramientas para realizar la programación de los trabajos son, según Duffuaa y otros (2010): orden de trabajo, plan de trabajo, acumulación de órdenes de trabajo pendientes, reportes de disponibilidad de mano de obra, reporte de disponibilidad de materiales, programa maestro de mantenimiento preventivo, programa de producción e índices históricos de terminación.

De lo expuesto el investigador deduce que, en la planeación del mantenimiento se considera la determinación de recursos necesarios para llevar adelante las funciones del mantenimiento, mientras que en la programación del mantenimiento se realiza un proceso de asignación de recursos y personal para los trabajos que tienen que realizarse en ciertos momentos. En esta etapa, se acoplan los trabajos que van a ejecutarse durante el mantenimiento y los recursos que serán empleados, asignando una secuencia que clarifique que serán llevados a cabo en ciertos puntos de tiempo.

\section{Ejecución}

En las fases anteriores se han fijado el punto de partida y los objetivos a los que se quiere llegar (planificación), de igual manera se determina la estructura de trabajo y las funciones dentro de la estructura (organización), asimismo se procede a la asignación de recursos y personal para los trabajos (programación). Seguidamente se procede a la ejecución del mantenimiento. En este aspecto, la ejecución es el momento en el 
cual se pone en práctica lo acordado en las fases de planificar, organizar y programar; es aquí donde los trabajadores se disponen a operar para alcanzar los objetivos previstos.

En el contexto del mantenimiento, Martínez (2007) expresa que la ejecución se refiere a la realización práctica de las actividades planificadas y programadas. Continúa el autor explicando que la ejecución del mantenimiento, al igual que el resto de las funciones administrativas, requiere de una formulación de objetivos y metas, planificación de actividades, programación de tareas, asignación de responsables y de los recursos a emplearse $y$, por último, la realización de las acciones de mantenimiento, así como la evaluación y control de los resultados que conlleven a tomar medidas correctivas.

Por su parte, Zambrano y Leal (2006) expresan que en la ejecución del mantenimiento se vinculan acciones administrativas con la dirección y coordinación de esfuerzos de los grupos de ejecución, tales esfuerzos son generados por la planeación y programación. Durante la ejecución, se siguen normas y procedimientos preestablecidos, a fin de lograr los objetivos del mantenimiento. Ahora bien, Márquez (2010) define la ejecución como la parte del proceso de la gestión en la cual se realizan los trabajos de mantenimiento, tanto programados como de emergencia, consiste en la realización del trabajo diario, que implica: suministro de materiales y equipos, seguridad del trabajo diario, medición, registro de datos, supervisión y seguimiento del trabajo diario.

Puede observarse, a juicio del investigador, como la ejecución es el momento (dentro del proceso de la gestión de mantenimiento), donde va a llevarse a cabo lo que fue previsto al planear el mantenimiento $y$, posteriormente, incluido en el programa de mantenimiento. Es importante acotar que para ejecutar el programa se requiere elaborar reportes de las actividades que se van a ejecutar, por lo general se utilizan las ordenes de trabajo, esto para facilitar el control.

\section{Control}

La gestión de mantenimiento se pone en movimiento por la demanda de trabajos de mantenimiento. En la carga de trabajo de este tipo, influye sobre todo la filosofía de mantenimiento. La administración y control del trabajo son esenciales para lograr los planes establecidos. El sistema de órdenes de trabajo es la herramienta que se utiliza para controlar el trabajo. Una orden de trabajo bien diseñada con un adecuado sistema de informes es el corazón del sistema de mantenimiento (Duffuaa y otros, 2010).

En este orden de ideas, Márquez (2010) indica que, el control es una parte esencial de la gestión de mantenimiento, involucra el control del trabajo, control de inventario, control de costos, y control de calidad a fin de garantizar el cumplimiento de los objetivos planeados. Este control es esencial, como sostiene este autor, para seguir logrando los planes de mantenimiento establecidos.

Ahora bien, Leal y Zambrano (2011) consideran que, el establecimiento de indicadores para el seguimiento y control de mantenimiento permiten verificar la fluidez del proceso y garantizar la adecuada gestión del sistema de mantenimiento en una organizaron o empresa. Algunos de los beneficios que tiene el establecimiento de los indicadores para la función mantenimiento son: 
Apoyo al proceso de planificación: (definición de objetivos y metas) y formulación de políticas de mediano y largo plazo.

$\checkmark$ Posibilita la detección de procesos en la empresa en los cuales existen problemas de gestión, tales como: uso ineficiente de recursos, demoras excesivas en la entrega del servicio al cliente, asignación del personal a las diferentes actividades.

$\checkmark$ La identificación de prioridades del cliente para el logro de los resultados deseados y así su satisfacción con el servicio ofrecido.

$\checkmark$ Seguimiento del proceso: el mejoramiento continuo solo es posible si se hace un seguimiento exhaustivo a cada eslabón de la cadena que conforma el proceso de mantenimiento. Las mediciones son las herramientas básicas no solo para detectar las oportunidades de mejora, sino además de para implementar las acciones.

$\checkmark$ Gerencia del cambio: un adecuado sistema de medición permite a las personas conocer su aporte a las metas organizacionales y cuáles son los resultados que soportan la afirmación de lo que está realizando bien.

Con base a lo anterior, a fines de la investigación, el control se enfoca en evaluar y corregir el desempeño de las actividades de mantenimiento de los subordinados para asegurar que los objetivos y planes de la organización se están llevando a cabo. La importancia del control radica en que en esta etapa es donde se logrará precisar si lo realizado se ajusta a lo planeado y, en caso de existir desviaciones, identificar los responsables y corregir dichos errores.

\section{Evaluación}

En la evaluación de un sistema de mantenimiento organizado se mide todo el conjunto en general que lo forma. En la gestión de mantenimiento, no solo se controla y evalúan los servicios prestados, sino al personal que constituye la unidad, herramientas, equipos, definiciones e instalaciones, y puede realizarse por medio analítico.

Según la Fundación Educación Industrias Fundei (2009), la gestión de mantenimiento no solo se controla por los movimientos de los servicios prestados, sino también puede medirse por índices que analizados conducen a la toma de decisiones. Estos índices son formulaciones basadas en el número de órdenes de trabajo, número de órdenes pendientes, avance de los trabajadores en ejecución y trabajos terminados, horashombres efectivas y no efectivas, eficiencia porcentual de cada sección.

Por su parte, Acosta (2007) indica que se debe evaluar la eficiencia de la política de mantenimiento que se ha planificado para el entorno productivo de una organización, e identificar las áreas en que tiene deficiencias estructurales y circunstanciales, lo cual es un aporte sustancioso puesto que la dirección puede establecer una ruta de acción para erradicar las debilidades detectadas.

Es por ello, que para el investigador, la aplicación periódica de instrumentos de evaluación permite detectar cómo responde la organización ante las variaciones a las que está constantemente sujeta y de qué forma debe adecuarse. Es por esto que hoy día las evaluaciones forman parte de revisiones cíclicas y sistemáticas, donde se identifican las desviaciones y se plantean soluciones a las mismas. 
MÉTODO

El tipo de investigación utilizado en este estudio, se definió como descriptivo, con un diseño no experimental, transeccional y de campo. La población quedó conformada por los gerentes, líderes y supervisores de las empresas que realizan el mantenimiento a las estructuras metálicas de la industria petrolera en el occidente de Venezuela, siendo éstas: IMDISMA C.A. SERTEJERCA y ANCRI RADIADORES C.A.

Para la recolección de datos se aplicó la técnica de la encuesta, a través del uso de un cuestionario contentivo de 30 ítems, utilizando una escala de frecuencia. La validación del instrumento se realizó mediante el juicio de cinco expertos. La confiabilidad se calculó a través del coeficiente Alfa de Cronbach, cuyo resultado fue de 0,82, mostrando muy alta confiabilidad. El análisis de los resultados se realizó a través de las frecuencias con base a los baremos diseñados mostrado en la Tabla 1.

Tabla 1. Categoría de análisis para la frecuencia

\begin{tabular}{cll}
\hline Alternativa & Categoría & \multicolumn{1}{c}{ Interpretación } \\
\hline $\mathrm{S}+\mathrm{CS} \geq 75 \%$ & Alta presencia & $\begin{array}{l}\text { Indica alta fortaleza para las etapas del proceso de } \\
\text { gestión de mantenimiento }\end{array}$ \\
$45 \% \leq \mathrm{S}+\mathrm{CS}<75 \%$ & Moderada presencia & $\begin{array}{l}\text { Indica leve fortaleza para las etapas del proceso de } \\
\text { gestión de mantenimiento }\end{array}$ \\
$\mathrm{S}+\mathrm{CS}<45 \%$ & Baja presencia & $\begin{array}{l}\text { Indica una debilidad para las etapas del proceso } \\
\text { de gestión de mantenimiento }\end{array}$ \\
\hline
\end{tabular}

Fuente: Elaboración propia

RESULTADOS

Como puede observarse en la Tabla 2, un $90,48 \%$ en promedio de los encuestados respondieron a los ítems involucrados en las alternativas siempre y casi siempre, indicando que la planificación, como etapa del proceso de la gestión de mantenimiento, tiene alta presencia, esto implica alta fortaleza de la gestión de mantenimiento en las empresas bajo estudio.

De acuerdo a los valores obtenidos por ítem, se observa que, todos arriban a la categoría de alta presencia indicando altas fortalezas para la gestión de mantenimiento en las empresas estudiadas, según el baremo diseñado y las respuestas dadas por los encuestados, de manera que en estas empresas se ejecuta con alta frecuencia lo referente a si: realizan la planificación del mantenimiento con holgura del tiempo de ejecución (90,48\%); en la planificación, determinan el personal requerido para la ejecución del mantenimiento $(95,24 \%)$; antes de iniciar el mantenimiento, se determinan los materiales que se requieren para llevarlo a cabo (90,48\%); previa a la ejecución del mantenimiento se determinan las herramientas requeridas para realizarlo $(90,48 \%) ; y$ en el plan se definen las metas que se desean alcanzar $(85,71 \%)$. 
Tabla 2. Indicador: Planificación

Nro. En la empresa donde usted labora:

1. Realizan la planificación del mantenimiento con holgura del tiempo de ejecución.

2. En la planificación, determinan el personal requerido para la ejecución del mantenimiento

3. Antes de iniciar el mantenimiento, se determinan los materiales que se requieren para llevarlo a cabo.

4. Previa a la ejecución del mantenimiento se determinan las herramientas requeridas para realizarlo.

5. En el plan se definen las metas que se desean alcanzar.

\begin{tabular}{|c|c|c|c|c|c|c|c|c|c|c|}
\hline \multirow{3}{*}{$\begin{array}{l}\text { Opción } \\
\text { Siemnre }\end{array}$} & \multicolumn{2}{|c|}{1} & \multicolumn{2}{|c|}{2} & \multicolumn{2}{|c|}{3} & \multicolumn{2}{|c|}{4} & \multicolumn{2}{|c|}{5} \\
\hline & $\mathrm{Fa}$ & $\mathrm{Fr}$ & $\mathrm{Fa}$ & $\mathrm{Fr}$ & $\mathrm{Fa}$ & $\mathrm{Fr}$ & $\mathrm{Fa}$ & $\mathrm{Fr}$ & $\mathrm{Fa}$ & $\mathrm{Fr}$ \\
\hline & 5 & 23,81 & 11 & 52,38 & 11 & 52,38 & 10 & 47,62 & 10 & 47,62 \\
\hline Casi siempre & 14 & 66,67 & 9 & 42,86 & 8 & 38,10 & 9 & 42,86 & 8 & 38,10 \\
\hline Algunas veces & 2 & 9,52 & 1 & 4,76 & 2 & 9,52 & 2 & 9,52 & 3 & 14,29 \\
\hline Casi nunca & 0 & 0,00 & 0 & 0,00 & 0 & 0,00 & 0 & 0,00 & 0 & 0,00 \\
\hline Nunca & 0 & 0,00 & 0 & 0,00 & 0 & 0,00 & 0 & 0,00 & 0 & 0,00 \\
\hline Total & 21 & 100 & 21 & 100 & 21 & 100 & 21 & 100 & 21 & 100 \\
\hline $\mathrm{S}+\mathrm{Cs}$ & \multicolumn{2}{|c|}{$90,48 \%$} & \multicolumn{2}{|c|}{$95,24 \%$} & \multicolumn{2}{|c|}{$90,48 \%$} & \multicolumn{2}{|c|}{$90,48 \%$} & \multicolumn{2}{|c|}{$85,71 \%$} \\
\hline Categoría & \multicolumn{2}{|c|}{ Alta presencia } & \multicolumn{2}{|c|}{ Alta presencia } & \multicolumn{2}{|c|}{ Alta presencia } & \multicolumn{2}{|c|}{$\begin{array}{c}\text { Alta } \\
\text { presencia }\end{array}$} & \multicolumn{2}{|c|}{ Alta presencia } \\
\hline $\begin{array}{l}\text { Descripción } \\
\text { Indicador } \\
\text { Categoría } \\
\text { Descripción }\end{array}$ & \multicolumn{2}{|c|}{ Alta fortaleza } & \multicolumn{2}{|c|}{ Alta fortaleza } & \multicolumn{2}{|c|}{$\begin{array}{c}\text { Alta fortaleza } \\
90,48 \% \\
\text { Alta presencia } \\
\text { Alta fortaleza }\end{array}$} & \multicolumn{2}{|c|}{ Alta fortaleza } & \multicolumn{2}{|c|}{ Alta fortaleza } \\
\hline
\end{tabular}

Fuente: Elaboración propia

Lo mostrado, en cuanto a la categoría de alta presencia de la planificación en la gestión de mantenimiento, coincide en alto grado con lo expuesto por Leal y Zambrano (2011), para quienes, en el contexto específico del mantenimiento, la planificación está referida a determinar los recursos necesarios para satisfacer la demanda de trabajos a los que va a responder el mantenimiento; es el proceso a través del cual se determinan qué elementos serán necesarios para realizar una tarea de mantenimiento, antes que se dé inicio al trabajo. Entre los recursos necesarios, se incluyen la fuerza laboral, los repuestos, el equipo y las herramientas.

Con base a lo descrito en estos resultados, para el investigador, en las empresas que realizan el mantenimiento a las estructuras metálicas se maneja la planificación con un alto criterio como etapa clave del proceso de gestión de mantenimiento, lo cual indica que aquellos aspectos relacionados a la acción de planificar el mantenimiento son tratados con alta presencia, otorgándoles así a estas empresas una alta fortaleza en su gestión. De tal forma, se valida su posición, cuando afirma que, planear es la base para poder ejecutar las acciones de mantenimiento, sin importar de que tipo sea, así tener de una manera ordenada los pasos a seguir para que se cumpla el trabajo en sinergia.

En lo concerniente al indicador organización, se observa, en la Tabla 2, un promedio de frecuencias de $84,76 \%$, indicando alta presencia de este indicador, como etapa del proceso de la gestión de 
mantenimiento, en las empresas analizadas, implicando una alta fortaleza según el baremo diseñado. Al detalle se observa que, la suma de las opciones positivas, siempre y casi siempre, para todos los reactivos aplicados se sitúa en alta presencia, implicando altas fortalezas para las empresas estudiadas.

Tabla 3. Indicador: Organización

Nro. En la empresa donde usted labora:

6. Las funciones que realiza el personal de mantenimiento están claramente diferenciadas según el cargo que ocupa

7. Las responsabilidades del personal de mantenimiento son asignadas de tal manera que no existe solapamiento.

8. La cantidad de subordinados para cada líder permite un control eficiente del trabajo de mantenimiento realizado.

9. Establecen descripciones de cargos para el personal de mantenimiento.

10. El mantenimiento se estructura de manera que se dé respuesta oportuna a las necesidades.

\begin{tabular}{|c|c|c|c|c|c|c|c|c|c|c|}
\hline \multirow{2}{*}{$\begin{array}{l}\text { Optems } \\
\text { Opción } \\
\text { Siempre }\end{array}$} & \multicolumn{2}{|c|}{1} & \multicolumn{2}{|c|}{2} & \multicolumn{2}{|c|}{3} & \multicolumn{2}{|c|}{4} & \multicolumn{2}{|c|}{5} \\
\hline & $\begin{array}{l}\mathrm{Fa} \\
11\end{array}$ & $\begin{array}{c}\mathrm{Fr} \\
52,38\end{array}$ & $\begin{array}{c}\mathrm{Fa} \\
5\end{array}$ & $\begin{array}{c}\mathrm{Fr} \\
23,81\end{array}$ & $\begin{array}{c}\mathrm{Fa} \\
8\end{array}$ & $\begin{array}{c}\mathrm{Fr} \\
38,10\end{array}$ & $\begin{array}{l}\mathrm{Fa} \\
12\end{array}$ & $\begin{array}{c}\mathrm{Fr} \\
57,14\end{array}$ & $\begin{array}{c}\mathrm{Fa} \\
5\end{array}$ & $\begin{array}{c}\mathrm{Fr} \\
23,81\end{array}$ \\
\hline Casi siempre & 7 & 33,33 & 10 & 47,62 & 11 & 52,38 & 6 & 28,57 & 14 & 66,67 \\
\hline Algunas veces & 3 & 14,29 & 5 & 23,81 & 2 & 9,52 & 3 & 14,29 & 2 & 9,52 \\
\hline Casi nunca & 0 & 0,00 & 1 & 4,76 & 0 & 0,00 & 0 & 0,00 & 0 & 0,00 \\
\hline Nunca & 0 & 0,00 & 0 & 0,00 & 0 & 0,00 & 0 & 0,00 & 0 & 0,00 \\
\hline Total & 21 & 100 & 21 & 100 & 21 & 100 & 21 & 100 & 21 & 100 \\
\hline $\begin{array}{l}\text { S + Cs } \\
\text { Categoría } \\
\text { Descripción } \\
\text { Indicador } \\
\text { Categoría } \\
\text { Descripción }\end{array}$ & \multicolumn{2}{|c|}{$\begin{array}{c}85,71 \% \\
\text { Alta presencia } \\
\text { Alta fortaleza }\end{array}$} & \multicolumn{2}{|c|}{$\begin{array}{c}71,43 \% \\
\text { Alta presencia } \\
\text { Alta fortaleza }\end{array}$} & \multicolumn{2}{|c|}{$\begin{array}{c}90,48 \% \\
\text { Alta presencia } \\
\text { Alta fortaleza } \\
84,76 \% \\
\text { Alta presencia } \\
\text { Alta fortaleza }\end{array}$} & \multicolumn{2}{|c|}{$\begin{array}{l}85,71 \% \\
\text { Alta presencia } \\
\text { Alta fortaleza }\end{array}$} & \multicolumn{2}{|c|}{$\begin{array}{c}90,48 \% \\
\text { Alta presencia } \\
\text { Alta fortaleza }\end{array}$} \\
\hline
\end{tabular}

Fuente: Elaboración propia

Así, el instrumento diseñado arrojó que en estas empresas: las funciones que realiza el personal de mantenimiento están claramente diferenciadas según el cargo que ocupa $(85,71)$; las responsabilidades del personal de mantenimiento son asignadas de tal manera que no existe solapamiento $(71,43 \%)$; la cantidad de subordinados para cada líder permite un control eficiente del trabajo de mantenimiento realizado (90,48\%); establecen descripciones de cargos para el personal de mantenimiento $(85,71 \%)$; y el mantenimiento se estructura de manera que se dé respuesta oportuna a las necesidades $(90,48 \%)$.

Lo mostrado valida, en alta medida, lo postulado por García (2009) cuando afirma que, la organización es la etapa de la gestión donde se debe determinar la estructura de trabajo, las funciones dentro de la estructura, las relaciones externas e internas, los procedimientos para el flujo y registro de información, así como la preparación de todos los recursos involucrados en el proceso: 
materiales, humanos, tecnológicos, entre otros.

Para el investigador es evidente, de acuerdo a lo mostrado, que en las empresas bajo estudio, se considera en alta medida a la organización como etapa del proceso de la gestión de mantenimiento, otorgándole alta presencia por parte de los encuestados, lo que se traduce en una alta fortaleza para esta gestión. Validándolo cuando afirma que, la organización, en la gestión de mantenimiento, es la fase de procura y distribución de responsabilidades, fijación de tiempo, esfuerzo, costo, necesarios para cada una de las actividades planificadas.

De seguido, en la tabla 4 se resume el comportamiento para el indicador programación, donde se observa que alcanzó un promedio de frecuencias de $74,29 \%$, indicando alta presencia del mismo como etapa del proceso de la gestión de mantenimiento en las empresas bajo estudio, implicando a su vez una alta fortaleza para dichas empresas. De manera específica, puede observarse como todos los ítems arriban a la categoría de alta presencia, a excepción del reactivo 12 catalogado con mediana presencia.

Así entonces, los ítems medidos se convierten en altas fortalezas para estas empresas, al otorgar alta presencia en cuanto a si: aplican programas de trabajo para ejecutar el mantenimiento (76,19\%); el programa de trabajo incluye la asignación de recursos (80,95\%); el programa de mantenimiento contempla las prioridades de los equipos o de la situación requerida de mantenimiento $(85,71 \%) ; y$ asignan los recursos disponibles para que los trabajos se realicen eficientemente en el tiempo planeado (80,95\%). Mientras, califican con mediana presencia, por ende es una leve fortaleza, el que utilicen un software para efectuar la programación de mantenimiento $(47,62 \%)$.

Tabla 4. Indicador: Programación

Nro. En la empresa donde usted labora:

11. Aplican programas de trabajo para ejecutar el mantenimiento.

12. Utiliza un software computarizado para efectuar la programación de mantenimiento.

13. En el programa de trabajo se incluye la asignación de recursos.

14. El programa de mantenimiento contempla las prioridades de los equipos o de la situación requerida de mantenimiento.

15. Asignan los recursos disponibles para que los trabajos se realicen eficientemente en el tiempo planeado. 
Nro. En la empresa donde usted labora:

\begin{tabular}{|c|c|c|c|c|c|c|c|c|c|c|}
\hline \multirow{5}{*}{$\begin{array}{l}\text { Opción } \\
\text { Siemprems } \\
\text { Casi siempre } \\
\text { Algunas veces }\end{array}$} & \multicolumn{2}{|c|}{1} & \multicolumn{2}{|c|}{2} & \multicolumn{2}{|c|}{3} & \multicolumn{2}{|c|}{4} & \multicolumn{2}{|c|}{5} \\
\hline & $\mathrm{Fa}$ & $\mathrm{Fr}$ & $\mathrm{Fa}$ & $\mathrm{Fr}$ & $\mathrm{Fa}$ & $\mathrm{Fr}$ & $\mathrm{Fa}$ & $\mathrm{Fr}$ & $\mathrm{Fa}$ & $\mathrm{Fr}$ \\
\hline & 5 & 23,81 & 4 & 19,05 & 5 & 23,81 & 9 & 42,86 & 4 & 19,05 \\
\hline & 11 & 52,38 & 6 & 28,57 & 12 & 57,14 & 9 & 42,86 & 13 & 61,90 \\
\hline & 5 & 23,81 & 3 & 14,29 & 3 & 14,29 & 3 & 14,29 & 4 & 19,05 \\
\hline Casi nunca & 0 & 0,00 & 7 & 33,33 & 1 & 4,76 & 0 & 0,00 & 0 & 0,00 \\
\hline Nunca & 0 & 0,00 & 1 & 4,76 & 0 & 0,00 & 0 & 0,00 & 0 & 0,00 \\
\hline Total & 21 & 100 & 21 & 100 & 21 & 100 & 21 & 100 & 21 & 100 \\
\hline $\mathrm{S}+\mathrm{Cs}$ & \multicolumn{2}{|c|}{$76,19 \%$} & \multicolumn{2}{|c|}{$47,62 \%$} & \multicolumn{2}{|c|}{$80,95 \%$} & \multicolumn{2}{|c|}{$85,71 \%$} & \multicolumn{2}{|c|}{$80,95 \%$} \\
\hline Categoría & \multicolumn{2}{|c|}{ Alta presencia } & \multirow{2}{*}{\multicolumn{2}{|c|}{$\begin{array}{c}\text { Mediana } \\
\text { presencia } \\
\text { Leve fortaleza }\end{array}$}} & \multicolumn{2}{|c|}{ Alta presencia } & \multicolumn{2}{|c|}{ Alta presencia } & \multicolumn{2}{|c|}{ Alta presencia } \\
\hline $\begin{array}{l}\text { Descripción } \\
\text { Indicador } \\
\text { Categoría } \\
\text { Descripción }\end{array}$ & \multicolumn{2}{|c|}{ Alta fortaleza } & & & \multicolumn{2}{|c|}{$\begin{array}{c}\text { Alta fortaleza } \\
74,29 \% \\
\text { Alta presencio } \\
\text { Alta fortaleza }\end{array}$} & \multicolumn{2}{|c|}{ Alta fortaleza } & \multicolumn{2}{|c|}{ Alta fortaleza } \\
\hline
\end{tabular}

Fuente: Elaboración propia

Los resultados validan, en alta medida, lo postulado por Leal y Zambrano (2011), para

quienes la programación del trabajo encomienda al personal de mantenimiento las asignaciones específicas de trabajo, con la suficiente anticipación como para garantizar la máxima coordinación entre mano de obra, material y equipo. La programación es un plan de acción cuidadosamente preparado que ha considerado la disponibilidad de mano de obra, materiales, equipos y las prioridades relativas de los trabajos. Es un procedimiento que inicia con los trabajos que tengan la prioridad más alta en la enumeración de pendientes.

Ahora bien, relacionando la teoría con la práctica, uno de los objetivos principales de las empresas prestatarias del servicio de mantenimiento debe ser generar en sus clientes la percepción de fiabilidad o responsabilidad, mostrando capacidad para desarrollar el servicio que se promete de forma digna, segura y precisa. Al desarrollar programas de mantenimiento, éstas deben hacer esfuerzos para demostrar a sus clientes que sus servicios ofrecidos garantizan un $\%$ o grado de confianza sobre los resultados futuros. Aspecto éste catalogado con alta presencia, implicando una alta fortaleza.

A continuación, en la Tabla 5, se muestran los resultados que corresponden al indicador ejecución, perteneciente a la dimensión que se analiza, se evidencia para el mismo un promedio de frecuencia de $83,81 \%$, indicando alta presencia de la ejecución como etapa del proceso de la gestión de mantenimiento en las empresas bajo estudio, implicando a su vez una alta fortaleza para dichas empresas, según el baremo utilizado. Del mismo modo, se puede observar como todas las actividades medidas, a través de los reactivos aplicados, fueron categorizadas con alta presencia, confiriéndoles altas fortalezas a las empresas en estos aspectos medidos. 
Tabla 5. Indicador: Ejecución

Nro. En la empresa donde usted labora:

16. El recurso humano asignado para ejecutar cada mantenimiento en el tiempo requerido, es suficiente.

17. Asignan recurso humano calificado para ejecutar cada mantenimiento

18. Las actividades de mantenimiento se ejecutan según un orden de prioridad establecido.

19. En la ejecución de los mantenimientos se cumple con las metas planificadas.

20. Al ejecutar las actividades de mantenimiento se siguen normas preestablecidas.

\begin{tabular}{|c|c|c|c|c|c|c|c|c|c|c|}
\hline Ítems & \multicolumn{2}{|c|}{1} & \multicolumn{2}{|c|}{2} & \multicolumn{2}{|c|}{3} & \multicolumn{2}{|c|}{4} & \multicolumn{2}{|c|}{5} \\
\hline $\begin{array}{l}\text { Opción } \\
\text { Siempre }\end{array}$ & $\begin{array}{r}\mathrm{Fa} \\
6\end{array}$ & $\begin{array}{c}\mathrm{Fr} \\
28,57\end{array}$ & $\begin{array}{l}\mathrm{Fa} \\
12\end{array}$ & $\begin{array}{c}\mathrm{Fr} \\
57,14\end{array}$ & $\begin{array}{c}\mathrm{Fa} \\
9\end{array}$ & $\begin{array}{c}\mathrm{Fr} \\
42,86\end{array}$ & $\begin{array}{c}\mathrm{Fa} \\
5\end{array}$ & $\begin{array}{c}\mathrm{Fr} \\
23,81\end{array}$ & $\begin{array}{c}\mathrm{Fa} \\
8\end{array}$ & $\begin{array}{c}\mathrm{Fr} \\
38,10\end{array}$ \\
\hline Casi siempre & 12 & 57,14 & 6 & 28,57 & 9 & 42,86 & 13 & 61,90 & 8 & 38,10 \\
\hline Algunas veces & 3 & 14,29 & 3 & 14,29 & 3 & 14,29 & 2 & 9,52 & 4 & 19,05 \\
\hline Casi nunca & 0 & 0,00 & 0 & 0,00 & 0 & 0,00 & 1 & 4,76 & 1 & 4,76 \\
\hline Nunca & 0 & 0,00 & 0 & 0,00 & 0 & 0,00 & 0 & 0,00 & 0 & 0,00 \\
\hline Total & 21 & 100 & 21 & 100 & 21 & 100 & 21 & 100 & 21 & 100 \\
\hline $\begin{array}{l}\text { S + Cs } \\
\text { Categoría } \\
\text { Descripción } \\
\text { Indicador } \\
\text { Categoría } \\
\text { Descripción }\end{array}$ & \multicolumn{2}{|c|}{$\begin{array}{c}85,71 \% \\
\text { Alta presencia } \\
\text { Alta fortaleza }\end{array}$} & \multicolumn{2}{|c|}{$\begin{array}{c}85,71 \% \\
\text { Alta presencia } \\
\text { Alta fortaleza }\end{array}$} & \multicolumn{2}{|c|}{$\begin{array}{c}85,71 \% \\
\text { Alta presencia } \\
\text { Alta fortaleza } \\
83,81 \% \\
\text { Alta presencia } \\
\text { Alta fortaleza }\end{array}$} & \multicolumn{2}{|c|}{$\begin{array}{l}85,71 \% \\
\text { Alta presencia } \\
\text { Alta fortaleza }\end{array}$} & \multicolumn{2}{|c|}{$\begin{array}{c}76,19 \% \\
\text { Alta presencio } \\
\text { Alta fortaleza }\end{array}$} \\
\hline
\end{tabular}

Fuente: Elaboración propia

Así, para un $85,71 \%$ se realiza con alta frecuencia lo referente a: el recurso humano asignado es suficiente para ejecutar cada mantenimiento en el tiempo requerido; asignan recurso humano calificado para ejecutar cada mantenimiento; las actividades de mantenimiento se ejecutan según un orden de prioridad establecido; en la ejecución del mantenimiento se cumple con las metas planificadas; mientras, para un $76,19 \%$ al ejecutar las actividades de mantenimiento se siguen normas preestablecidas.

Estos resultados son altamente coincidentes con lo manejado por Zambrano y Leal (2006), quienes expresan que en la ejecución del mantenimiento se vinculan acciones administrativas con la dirección y coordinación de esfuerzos de los grupos de ejecución, tales esfuerzos son generados por la planeación y programación. Durante la ejecución, se siguen normas preestablecidas, a fin de lograr los objetivos del mantenimiento.

En el caso de las empresas analizadas, se evidencia alta presencia de la ejecución como etapa del proceso de la gestión de mantenimiento que realizan, lo cual les confiere una alta fortaleza de acuerdo al baremo diseñado. Cabe resaltar una alta congruencia con el investigador, para quien la ejecución es el momento (dentro del proceso de la gestión de mantenimiento), donde se lleva a cabo lo que fue previsto al planear el mantenimiento $y$, posteriormente, incluido en el programa de mantenimiento.

Continuando la discusión de los resultados, en la Tabla 6 se presentan los logrados para el indicador control de la dimensión etapas del proceso de gestión, el 
cual obtuvo un promedio de frecuencia de $77,14 \%$, indicando que, de acuerdo a la percepción de los encuestados, la etapa control dentro del proceso de gestión de mantenimiento realizado por las empresas estudiadas, demuestra alta presencia respecto a la manera como se realiza el mismo, implicando a su vez una alta fortaleza para dichas empresas, según el baremo utilizado. Esta situación es explicada porque, todas las actividades medidas a través de los ítems aplicados fueron categorizadas con alta presencia, confiriéndoles altas fortalezas a las empresas en estos aspectos.

De forma tal que, en las empresas, se realiza con alta frecuencia lo concerniente a: para el seguimiento de los trabajos de mantenimiento se utilizan las órdenes de trabajo como sistema de control $(85,71 \%)$; comparan el porcentaje (\%) de mantenimiento realizado con el programado $(71,43 \%)$; analizan la información registrada sobre los mantenimientos realizados para detectar acciones correctivas (76,19\%); revisan periódicamente que los costos de mantenimiento estén dentro de lo previsto $(71,43 \%) ; y$ verifican que se dispone de inventarios de materiales, repuestos o piezas para ejecutar el mantenimiento $(80,95 \%)$. Estos resultados validan los postulados planteados en las bases teóricas de la investigación.

De tal forma, validan a Márquez (2010) cuando indica que, el control es una parte esencial de la gestión de mantenimiento, involucra el control del trabajo, control de inventario, control de costos, y control de calidad a fin de garantizar el cumplimiento de los objetivos planeados. Este control es esencial, como sostiene este autor, para seguir logrando los planes de mantenimiento establecidos, es decir, la administración y control del trabajo son esenciales para lograr los planes establecidos.

Tabla 6. Indicador: Control

\footnotetext{
Nro. En la empresa donde usted labora:

21. Para el seguimiento de los trabajos de mantenimiento se utilizan las órdenes de trabajo como sistema de control.

22. Comparan el \% de mantenimiento realizado con el programado

23. Analizan la información registrada sobre los mantenimientos realizados para detectar acciones correctivas.

24. Revisan periódicamente que los costos de mantenimiento estén dentro de lo previsto.

25. Verifican que se dispone de inventarios de materiales, repuestos o piezas para ejecutar el mantenimiento.
} 


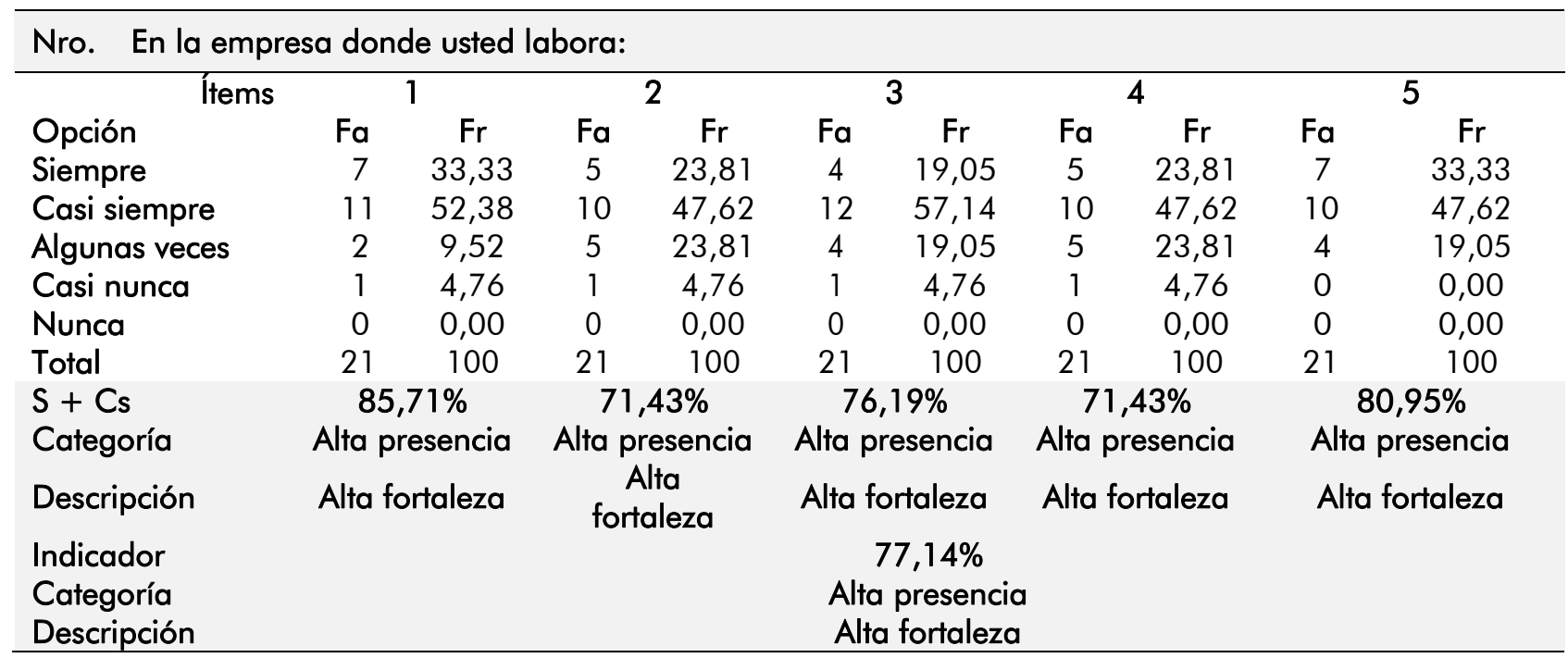

Fuente: Elaboración propia

De manera similar, se valida lo establecido por el investigador, para quien el control se enfoca en evaluar y corregir el desempeño de las actividades de mantenimiento de los subordinados para asegurar que los objetivos y planes de la empresa se están llevando a cabo. La importancia del control radica en que en esta etapa es donde se logrará precisar si lo realizado se ajusta a lo planeado $y$, en caso de existir desviaciones, identificar los responsables y corregir dichos errores. $Y$ para ello, el sistema de órdenes de trabajo es la herramienta clave que se utiliza para controlar el trabajo.

Ahora bien, en este orden de presentación, se tienen los resultados del último indicador considerado en la dimensión etapas del proceso de gestión; referido a la evaluación. En este sentido, la tabla 7 muestra un promedio de frecuencias de $75,24 \%$, indicando alta presencia de este indicador como etapa del proceso de gestión de mantenimiento realizado por las empresas bajo estudio, lo cual implica alta fortaleza.

Tabla 7. Indicador: Evaluación

\begin{tabular}{l} 
Nro. En la empresa donde usted labora: \\
\hline 26. Evalúan la eficiencia de la política de mantenimiento que se ha planificado \\
27. Identifican las áreas en que se tienen deficiencias circunstanciales. \\
28. La dirección establece rutas de acción para erradicar debilidades detectadas. \\
29. Evalúan el desempeño del personal que constituye la unidad de mantenimiento \\
30. Realizan evaluaciones cíclicas donde se identifiquen desviaciones para plantear soluciones a las \\
\end{tabular}




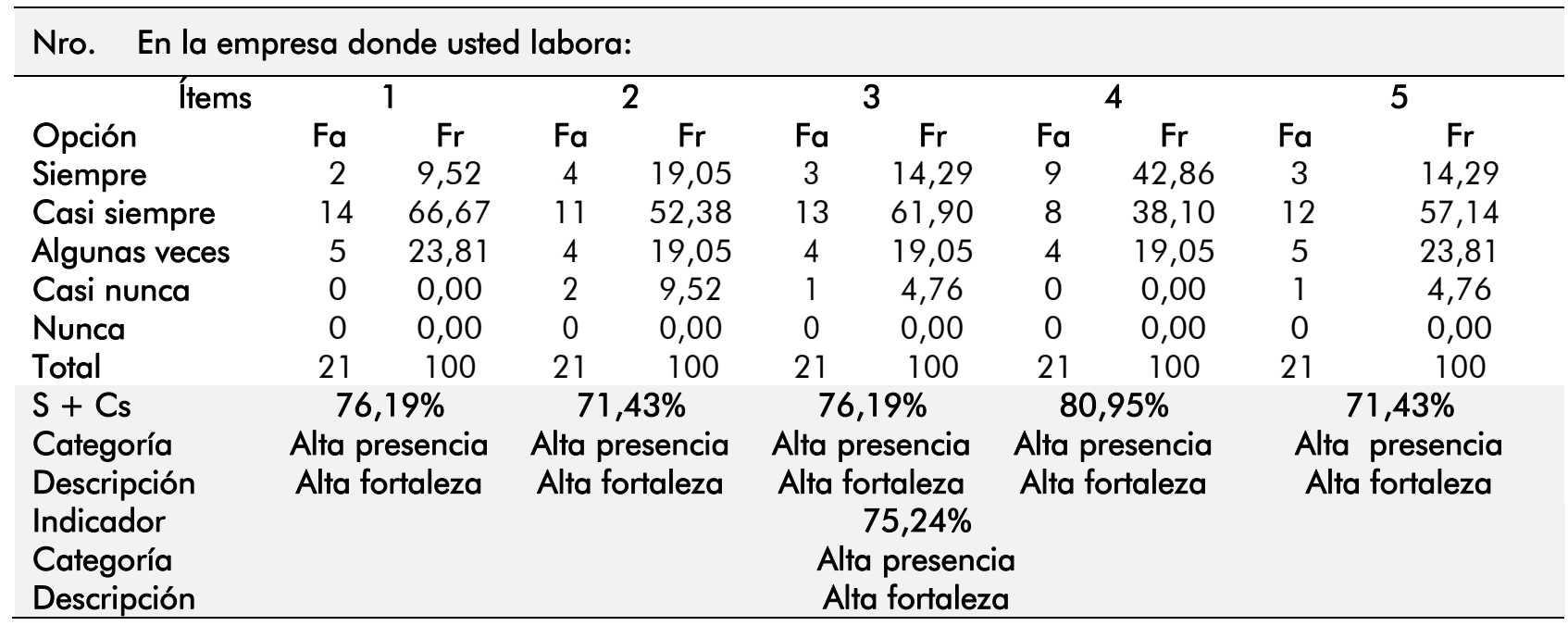

Fuente: Elaboración propia

Al desglosar resultados por ítems, se evidencia alta presencia para todos los reactivos medidos, confiriéndole a las empresas altas fortalezas en la evaluación de su gestión; de tal forma afirman realizar con alta frecuencia lo referido a: evalúan la eficiencia de la política de mantenimiento que se ha planificado $(76,19 \%)$; identifican las áreas en que se tienen deficiencias circunstanciales $(71,43 \%)$; la dirección establece rutas de acción para erradicar debilidades detectadas (76,19\%); evalúan el desempeño del personal que constituye la unidad de mantenimiento $(80,95 \%)$; realizan evaluaciones cíclicas donde se identifiquen desviaciones para plantear soluciones a las mismas $(71,43 \%)$.

Lo mostrado valida a Acosta (2007) cuando indica que, se debe evaluar la eficiencia de la política de mantenimiento que se ha planificado para el entorno productivo de una organización, e identificar las áreas en que tiene deficiencias circunstanciales $y$ estructurales, lo cual es un aporte sustancioso puesto que la dirección puede establecer una ruta de acción para erradicar las debilidades detectadas.

Visto así, se verifica nuevamente, como se manifestó en los indicadores anteriores, que las empresas sujetas a estudio valoran en alta medida las etapas del proceso de la gestión de mantenimiento, al otorgarles alta presencia en la realización del mismo, en este caso específico en lo que concierne a la evaluación que deben encarar los gerentes en su desempeño. Lo expuesto, valida al investigador cuando afirma que, la aplicación periódica de los instrumentos de evaluación permite detectar cómo responde la organización ante las variaciones a las que está constantemente sujeta y de qué forma debe adecuarse.

Luego de analizados los resultados obtenidos para cada uno de los indicadores que conforman la dimensión etapas del proceso de la gestión, en la tabla 8 se reflejan los resultados alcanzados para dicha dimensión. Se observa que todos los indicadores, aplicados para medir la dimensión, se posicionaron en el rango 
donde la suma de las opciones siempre y casi siempre son $\geq 70 \%$, indicando alta presencia del indicador con implicación de alta fortaleza, lo cual determinó un promedio de frecuencias para la dimensión de 80,95\%, bajo la interpretación del baremo como de alta presencia de la dimensión, implicando, a su vez, una alta fortaleza para las empresas analizadas.

Tabla 8. Dimensión: Ełapas del proceso de gestión

\begin{tabular}{|c|c|c|c|}
\hline INDICADOR & $S+C$ & RANGO & INTERPRETACIÓN \\
\hline Planificación & $90,48 \%$ & $S+C S \geq 70 \%$ & $\begin{array}{l}\text { Indica alta fortaleza para las etapas del proceso } \\
\text { de gestión de mantenimiento }\end{array}$ \\
\hline Organización & $84,76 \%$ & $S+C S \geq 70 \%$ & $\begin{array}{l}\text { Indica alta fortaleza para las etapas del proceso } \\
\text { de gestión de mantenimiento }\end{array}$ \\
\hline Programación & $74,29 \%$ & $S+C S \geq 70 \%$ & $\begin{array}{l}\text { Indica alta fortaleza para las etapas del proceso } \\
\text { de gestión de mantenimiento }\end{array}$ \\
\hline Ejecución & $83,81 \%$ & $S+C S \geq 70 \%$ & $\begin{array}{l}\text { Indica alta fortaleza para las etapas del proceso } \\
\text { de gestión de mantenimiento }\end{array}$ \\
\hline Control & $77,14 \%$ & $S+C S \geq 70 \%$ & $\begin{array}{l}\text { Indica alta fortaleza para las etapas del proceso } \\
\text { de gestión de mantenimiento }\end{array}$ \\
\hline Evaluación & $75,24 \%$ & $S+C S \geq 70 \%$ & $\begin{array}{l}\text { Indica alta fortaleza para las etapas del proceso } \\
\text { de gestión de mantenimiento }\end{array}$ \\
\hline DIMENSIÓN & $80,95 \%$ & $S+C S \geq 70 \%$ & $\begin{array}{l}\text { Indica alta fortaleza para las etapas del proceso } \\
\text { de gestión de mantenimiento }\end{array}$ \\
\hline
\end{tabular}

Fuente: Elaboración propia

Estos resultados, son coincidentes con lo postulado por Márquez (2010), para quien las etapas del proceso de la gestión de mantenimiento incluye el trabajo de planificar, organizar, programar, ejecutar, controlar y evaluar tomando en consideración los recursos, estas etapas deben realizarse para maximizar tanto la disponibilidad como efectividad de la infraestructura requerida por el sistema de producción. El propósito de estas etapas es mejorar la funcionalidad de los componentes de equipos o maquinarias, en función de la filosofía de las empresas, con la adopción de medidas, y la realización de acciones que permitan alcanzar los objetivos y la efectividad de sus procesos.
Al mismo tiempo validan, en alto grado, la posición de Martínez (2007) cuando afirma que, para cumplir las etapas de este proceso es prioritario definir un sistema de elementos con base a la dirección, que se relacione con la planificación, organización, programación, ejecución, control y evaluación. Así, la gestión de mantenimiento es un ciclo que se da en forma secuencial, cuyo proceso consta de seis (6) etapas, ya mencionadas, y este ciclo permite realizar una retroalimentación a fin de permitir una mejora continua.

Al detalle se observa como en las empresas que realizan el mantenimiento a las estructuras metálicas de la industria petrolera en occidente, las etapas del 
proceso de la gestión de mantenimiento consideradas: planificación, organización, programación, ejecución, control y evaluación, muestran alta presencia en los servicios prestados, según los encuestados, implicando que ellas poseen una alta fortaleza en la generación de los servicios de mantenimiento ofrecidos. Los mismos se constituyen en los indicadores que les permitirán la medición de la calidad del servicio que prestan.

Esta situación valida al investigador, para quien las etapas del proceso de la gestión de mantenimiento son el conjunto de acciones pertinentes para su cumplimiento, que contribuyan a la efectividad de los procesos productivos, y considerada dentro de la tendencia sistémica de la gestión de mantenimiento como: planificación, organización, programación, ejecución, control y evaluación, tomando en cuenta siempre los recursos.

\section{CONCLUSIONES}

Se describieron las etapas del proceso de la gestión de mantenimiento presentes en las empresas que realizan el mantenimiento a las estructuras metálicas de la industria petrolera en occidente, se concluye que estas organizaciones poseen alta presencia de estas etapas en su gestión, situación evidenciada en los niveles de presencia de cada una de ellas, donde la planificación organización, programación, ejecución, control y evaluación, se ubicaron en la categoría de alta presencia, confiriéndole altas fortalezas a la gestión llevada a cabo.

Sin embargo, se acota que aun con esta categoría de alta presencia alcanzada en las etapas del proceso de la gestión de mantenimiento, se detectó la existencia de ciertos aspectos a ser reforzados, específicamente en cuanto a la organización, programación control y evaluación. Estos aspectos muestran oportunidades de mejora, motivo por el cual la gerencia debe revisarlos, en virtud de la importancia que tienen estas etapas del proceso en la gestión de mantenimiento, debido a su efecto en toda la organización; de ello dependerá estrechamente el desarrollo de la gestión, a través del logro de sus objetivos.

\section{REFERENCIAS}

Acosta, H. (2007). El control y la evaluación de la gestión del mantenimiento. [Nota técnica en línea]. Ciudad de la Habana: Coordinación Editorial CEIM-CUJAE. Disponible en: http://www.cujae.edu.cu

Becerra, F. (2005). Gestión del Mantenimiento. Documento en línea. Disponible en: http://www.mantenimientomundial.com/s ites/mmnew/bib/notas/GestionBecerra.p $\mathrm{df}$

Clemenza, B. (2010). Como desarrollar e implantar un sistema de mantenimiento. Ediciones Astro Data. Maracaibo. Venezuela

Duffuaa, S.; Raouf, A; Campbell, J. (2010). Sistemas de Mantenimiento. Planificación y Control. Editorial Limusa. México

Fundación Educación Industrias (Fundei) (2009). Lagestión del mantenimiento. Material de curso. Venezuela

García, S. (2009). Organización y gestión integral de mantenimiento. Tercera edición. Ediciones Díaz de Santos S.A. Madrid, España

Jiménez, K. y Milano, T. (2006). Planificación y gestión del mantenimiento industrial. Un enfoque estratégico y operativo. Editorial Panapo. Caracas, Venezuela

Leal, S. y Zambrano, S. (2011). Índices e Indicadores de Gestión de Mantenimiento en las Pymes del Estado Táchira. 3er Congreso Uruguayo de Mantenimiento. Disponible 
en:http://www.uruman.org/3er_congreso docs/trabajos_tecnicos/Articulo\%20\%20 URU MAN\%20-\%20Indicadores.pdf

Márquez, M. (2010). Manual de la Ingeniería de Cali dad. Gestión de mantenimiento. Editorial Panapo. Caracas, Venezuela

Martínez, L. (2007). Organización y planificación de sistemas de mantenimiento. Centro de altos estudios gerenciales. Instituto Superior de Investigación y Desarrollo. 2da Edición. Caracas, Venezuela

Norma Venezolana COVENIN 3049-93. Mantenimiento. Definiciones. Comité
Técnico de Normalización CT-3 Construcción, aprobada por la COVENIN en su reunión № 124 de fecha 93-1201. Venezuela

Omeñaca, P. (2009). Aproximación de la gerencia de tecnología en la empresa. Papeles de trabajo del IESA. № 16. Segunda edición. Venezuela

Zambrano, S. y Leal, S. (2006). Manual práctico de gestión de mantenimiento. Fondo Editorial UNET. San Cristóbal, Venezuela 